\title{
CONTROL OF SECONDARY CROSSFLOW IN A HIGH-SPEED COMPRESSOR CASCADE BY ENDWALL FENCES WITH VARYING LOCATIONS
}

\author{
Yunzhi CHEN \\ School of Marine Engineering, \\ Dalian Maritime University \\ yunzhi_chen@dlmu.edu.cn \\ Dalian, Liaoning, China
}

\author{
Ling YANG \\ Merchant Marine College, \\ Shanghai Maritime University \\ yangling@shmtu.edu.cn \\ Shanghai, China
}

\author{
Jingjun ZHONG \\ Merchant Marine College, \\ Shanghai Maritime University \\ zhongjj@shmtu.edu.cn \\ Shanghai, China
}

\begin{abstract}
It is well known that the secondary flow, including vortexes, boundary layer, and three-dimensional separation, is detrimental to the efficiency and stability in the axial compressor. As a passive secondary flow control technique, the endwall fence is considered to overcome the adverse pressure gradient by blocking the crossflow. On this paper, a steady computational study is carried out in a high-speed compressor cascade. Two categories of fence configurations, i.e., embedded in the front and the back of flow passage, have been investigated. For each category, the fence position is selected at 7 locations ranging from $20 \%$ to $80 \%$ pitch length away from the blade pressure surface, the interval between each case is $10 \%$ pitch length. In the comparison between the datum and the fenced cascade, the case of $50 \%$ pitch length away from the blade suction surface in the category of front fences is most effective in the improvement of the aerodynamic performance. At the design point, this case decreases the loss coefficient and the blockage coefficient by $6.3 \%$ and $6.5 \%$, respectively. Meanwhile, it increases the absolute value of the flow turning angle by $0.9 \%$. From the perspective of the vortex structure, both optimum fences in the front and back category can suppress the passage vortex by inducing the counter-rotating fence vortex, of which scale has a relationship with the contact area between fence surface and the crossflow. Last but not least, the fenced cascade with an optimum performance both in loss mechanism and vortex structure has been investigated under varying inlet incidences. The comparison analysis of the baseline and optimum fence and demonstrates that there is a reduction of the loss exists at negative incidences while an augment occurs at positive incidences. In summary, the optimum case of endwall fence can effectively weaken the endwall loss and thereby enlarge the cascade operating range under negative incidences.
\end{abstract}

\section{INTRODUCTION}

The efficiency is probably the most crucial index for most turbomachines, which is influenced by some complex factors, such as profile loss, tip leakage loss, and endwall crossflow (Denton 1993). Over the past few decades, enormous efforts have been taken to reduce the endwall loss of different types of compressors. For modern high-speed compressors, both the higher transverse pressure gradient and the viscous effect may inevitably increase endwall loss, which carries the challenges to obtain further aerodynamic improvements (Gbadebo, Cumpsty, and Hynes 2005). Hence the endwall loss has a strong influence on overall cascade loss.

The flow control technology, acting on a small scale, is used to bring the desired behaviour change into a larger flow (Gad-el-Hak 1996). It is proved that flow control is effective in the sources of endwall flow such as the boundary separation, skin-friction, low momentum, and wake mixing. The mainly two types of flow control, active and passive, are witnessing the interest of market and research (Hecklau et al. 2012). This kind of classification for flow control methods considers the energy involved and the control loop included. Active control such as boundary layer suction (Zhang et al. 2018), synthetic jet (Zander et al. 2011), and plasma actuation ( $\mathrm{Li}$ et al. 2010), is widely researched depends on the recent advancement of computers and experiments. However, passive control is simpler to apply to an existing device and bring lower cost due to its simpler form.

Passive flow control of endwall loss is currently employed via shaping: endwall fence/groove (Hage and Paschereit 2007), vortex generator (Diaa et al. 2015), endwall contouring (Chen et al. 2012), casing treatment, etc, which is beneficial to delay or provoke separation. As a structural bulge along streamwise on endwall surface, endwall fence is available to hinder the crossflow and 
generate the fence vortex (Liu et al. 2017). In fact, the initiate of crossflow separation is induced as well as the mixing is enhanced under the positive effect of endwall fence. Yet, the negative characteristic is higher losses and thicker boundary layer in near-fence region.

Endwall fence technique has been utilized to reduce secondary flow loss in turbine cascades for a long history. Experimental investigations of (Kawai 1994) and Govardhan and Kumar (2011) found that fence with 1/3 height of the inlet boundary layer thickness when located half a pitch away from the blade suction side is optimal. Moreover, (Moon and Koh 2001) have simulated the complicated vortex structure of flow field in turbine cascade with and without endwall fence, which leads to an understanding on the formation of counter-rotating streamwise fence vortex and explains the mechanism of controlling secondary flow development. Based on these findings in the turbine, For loss reduction in a low-speed compressor, (Zhong et al. 2009) utilized experimental and numerical methods to investigate on the effect of the single streamwise endwall fence with various geometry parameters and pitchwise positions. He found that the optimal fence, $1 / 3$ height of boundary and $75 \%$ length of the chord, can reduce $22 \%$ of cascade total pressure loss when is located on $30 \%$ pitch away from the blade pressure surface. Move to the high-speed compressor, (Hergt et al. 2011) developed a series of non-axisymmetric endwall modification strategy, including a row of endwall fences with various heights. This strategy not only reduced the total loss of the cascade obviously in the aerodynamic design, but also showed the mechanism of endwall loss.

This article firstly focuses on the dependency of aerodynamic performance on the location of a single streamwise endwall fence under the design point, where the steady numerical study is conducted by means of ReynoldsAveraged Navier-Stokes approach. Then the detail of the flow field is discussed in order to understand the mechanism of flow control under a single streamwise endwall fence. The last but not the least, the comparison between the loss curve of the datum and the critical fenced cascades gives an indication on the further research.

\section{METHODOLOGY}

\section{Configurations of cascade}

The baseline linear configuration is a highly loaded compressor cascade with a NACA65-K48 profile (see Figure 1). The main geometrical and aerodynamic parameters of the cascade, as well as the test conditions in the aerodynamic design point, are shown in Table 1.

The commercial flow solver ANSYS CFX is utilized to solve the steady RANS equations to analyse flow phenomena in the endwall region in detail, the $\gamma-\theta$ transition model is employed in conjunction with the SST turbulence model, under $\mathrm{Ma}=0.7$. For all cases, a fine multi-block grid with an $\mathrm{H}-\mathrm{O}-\mathrm{H}$ topology, as shown in Figure 2, is densified to sufficiently resolve the blade and endwall boundary layer. The value of $y+$ adjacent to the wall is approximately 1.0, in order to meet the requirements of low Reynolds turbulence model.

Table 1 Main geometric parameters of profile

\begin{tabular}{llll}
\hline Parameter & symbol & Value & Unit \\
\hline Chord length & $\mathrm{C}$ & 60 & $\mathrm{~mm}$ \\
Span & $\mathrm{H}$ & 100 & $\mathrm{~mm}$ \\
Pitch & $\mathrm{T}$ & 33 & $\mathrm{~mm}$ \\
Inlet geometry angle & $\alpha$ & 48 & $\circ$ \\
Inflow Mach number & $\mathrm{Ma}_{\text {in }}$ & 0.7 & - \\
Inflow boundary layer thickness & $\delta$ & 13 & $\mathrm{~mm}$ \\
\hline
\end{tabular}

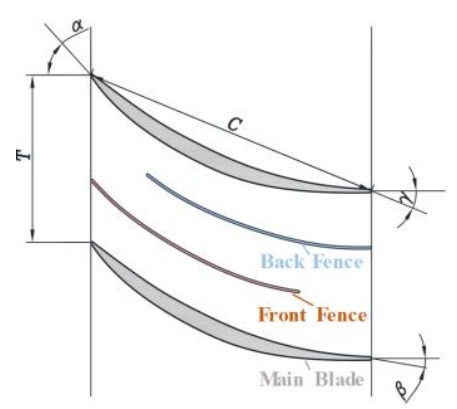

Figure 1 Diagram of the Cascade Geometry

\section{Numerical approach and validation with experiment}

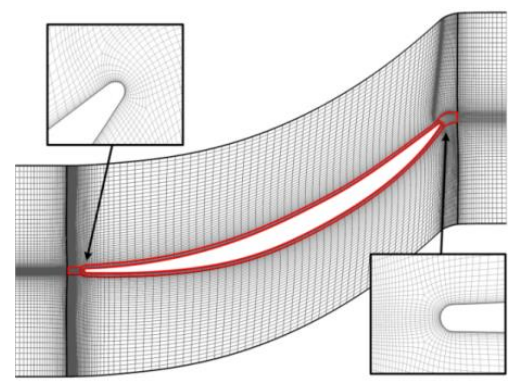

Figure 2 Typical mesh

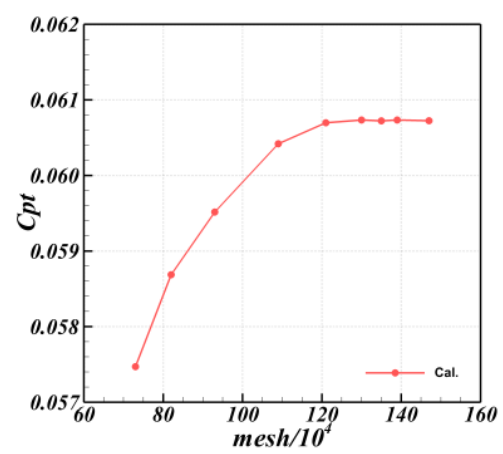

Figure 3 grid independence study

Numerical simulations are conducted with the same geometry and boundary conditions as those in the experimental condition. At the inlet, total pressure, total temperature $(310 \mathrm{~K})$, and flow angle $\left(-6^{\circ}\right)$ are obtained from validation experiments. At the outlet, the static pressure is specified. At the blade surface and hub, no-slip and adiabatic wall conditions are used. To reduce simulation cost, a single passage is cut down to a half span. Hence the top surface of 
computational domain is set to be a symmetric boundary, and both its sides set to be periodic boundary conditions.

The results of grid independence study are shown in Figure 3, where the grid of datum cascade consists of nearly 1.1 million nodes, and that of fenced cascade contains totally about 1.5 million nodes.

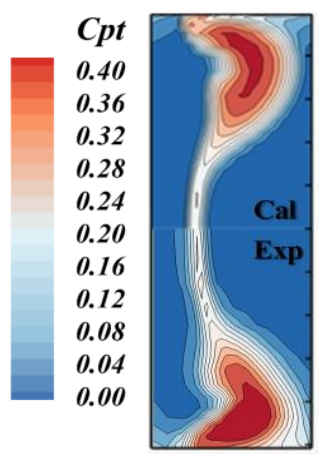

(a)

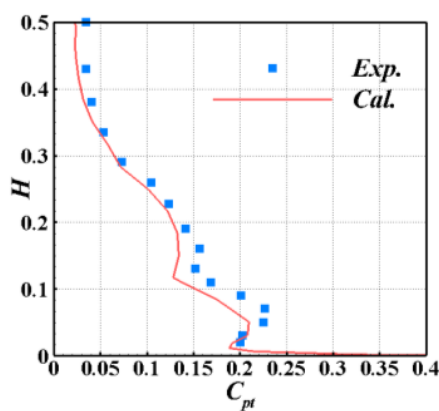

(b)

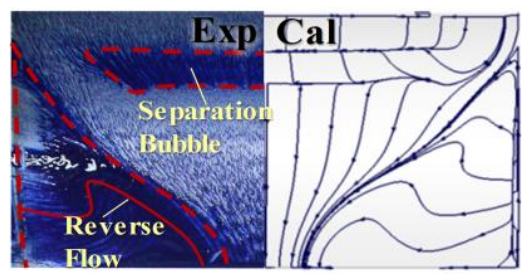

(c)

Figure 4 Validation of Computational Results:(a) Contours of the Total Pressure Loss; (b) Massaverage total pressure coefficient; (c) Numerical Limiting Streamlines and Experimental Oil Flow Visualization

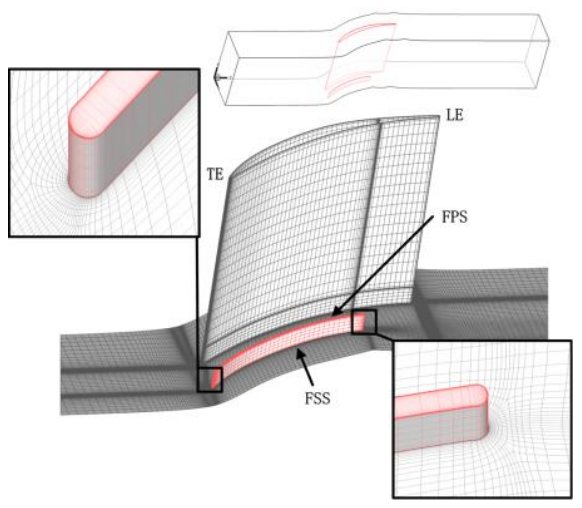

Figure 5 Distribution of Three-dimensional Computational Grid

Figure 4(a) shows the contours of total pressure loss at the exit of the cascade, i.e., $60 \% \mathrm{C}$ downstream the blade TE. Corresponding experimental results are obtained from a high-speed linear cascade wind tunnel of Dalian Maritime University. Although the calculated value is lower than experimental results in Figure 4(a) and 4(b), the distribution and tendency of $\mathrm{Cpt}$ is basically consistent. Moreover, Figure 4(c) shows the oil-flow visualization and the corresponding numerical limiting streamlines on the suction surface at $0^{\circ}$ incidence condition. In both simulation and experiment, the separation bubble exits at the $30 \% \mathrm{C}$, and the corner separation in the rear part of the blade is obviously wellmatched. These results illustrate that the calculated results have a good consistency with the experimental results both in aspects of quality and quantity.

Figure 5 presents the computational grid used in this study, the sculpt of fence is based on the rectangular sheet with rounding LE as well as TE, and parallel to the blade camber line. In order to explore the correlation between the endwall location of fence devices and the aerodynamic performance of cascades, fences in 14 cases with the same geometrical parameters $(5 \mathrm{~mm}$ in width, $75 \% \mathrm{C}$ in length and $10 \%$ inlet boundary thickness in height) are divided into two categories: FF and BF (see Figure 1). Table 2 details fenced locations, which is selected at 7 locations ranging from $20 \%$ to $80 \%$ pitch length away from the blade pressure surface. The interval between two adjacent fences is $10 \%$ pitch length.

Table 2 Main geometric parameters of profile

\begin{tabular}{llllllll}
\hline Categories & \multicolumn{7}{c}{ Distance from the SS (\% T) } \\
\hline FF cases & 20 & 30 & 40 & 50 & 60 & 70 & 80 \\
BF cases & 20 & 30 & 40 & 50 & 60 & 70 & 80 \\
\hline
\end{tabular}

\section{Calculation formulae}

The parameters used to quantificationally evaluate the aerodynamic performance of cascade are denoted as followed:

(1) Total pressure coefficient:

$$
C p t=\frac{P_{t, \text { in }}-P_{t, \text { out }}}{P_{t, \text { in }}-P_{\text {in }}} .
$$

(2) The total flow blockage coefficient in the passage:

$$
B=\frac{A_{b}}{A_{\text {out }}}=\frac{\iint\left(1-\frac{\rho V_{z}}{\rho_{e} V_{e}}\right) d A}{A_{\text {out }}} .
$$

(3) The pitch-averaged value of total pressure loss coefficient:

$$
\overline{C p t}=\frac{\int C p t d t}{d t}=\sum_{i=1}^{n}\left(C p t_{i} \Delta t_{i}\right) / t .
$$

(4) Axial vorticity:

$$
\Omega=\frac{\partial V x}{\partial y}-\frac{\partial V y}{\partial x}
$$

(5) The static pressure coefficient:

$$
C p=\frac{P_{\text {out }}-P_{\text {in }}}{P_{t, \text { in }}-P_{\text {in }}} .
$$

(6) The secondary flow in Cartesian coordinates of the linear cascade: 


$$
\begin{aligned}
& v_{s}=V \times \cos \beta \times \sin \left(\alpha_{s}\right) . \\
& \omega_{s}=V \times \sin \beta .
\end{aligned}
$$

\section{RESULTS AND DISCUSSION}

\section{Variations in aerodynamic performances of cascades}

Figure 6 shows the variations of three categories of cascades (the datum, FF, and BF) on general aerodynamic performance versus seven crosswise distances $(t)$, under the design point. On the whole, both the $50 \%$ cases of FF and BF are capable of reducing the total pressure loss and blockage coefficient at the same time. Furthermore, the mid-case of FF can mostly lower the outlet flow turning angle.

As shown in Figure 6(a) and 6(b), the total pressure loss and blockage coefficient shows a concave, which means the fences located medially have a positive effect on this cascade. In Figure 6(c), both the curves of turning angle in the series of FF and BF are fluctuating with the increasing distance between FSS and PS. Among the FF cases, the aerodynamic performance of $20 \%-50 \%$ cases is better, especially $40 \%$ and $50 \%$ cases: Cpt is singly reduced by $5.9 \%$ and $6.3 \%$; the $\mathrm{B}$ decreased $11.5 \%$ and $6.5 \%$; the $\beta$ slightly dropped $0.2 \%$ and $0.9 \%$. However, none of the BF cases can lower the three primary performance simultaneously. Only the $50 \%$ case declines the Cpt and B by $0.5 \%$ and $4.4 \%$.

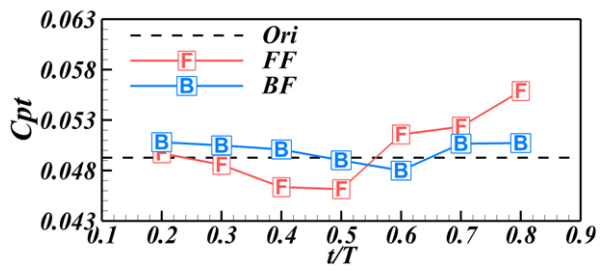

(a) Total Pressure Loss Coefficient

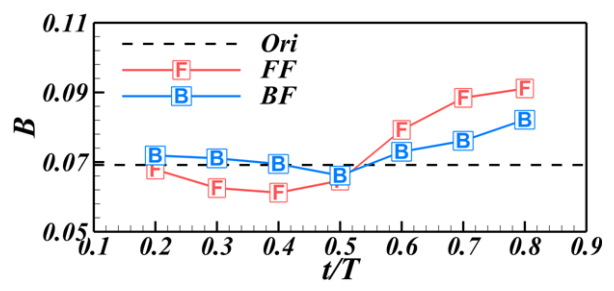

(b) Blockage Coefficient

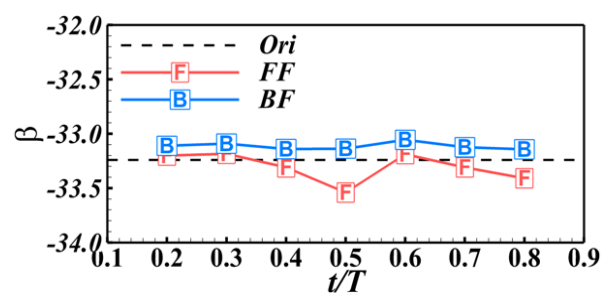

(c) Flow Turning Angle

Figure 6 Influence of Endwall Fence Locations on Main Aerodynamic Parameters

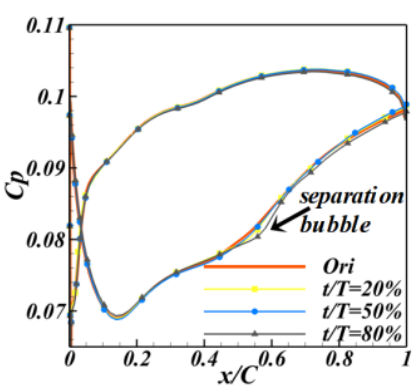

(a) $40 \% \mathrm{H}$ of $\mathrm{FF}$

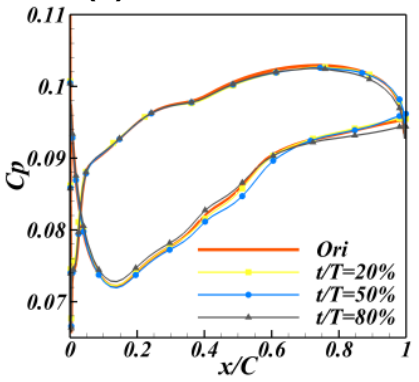

(c) $10 \% \mathrm{H}$ of $\mathrm{FF}$

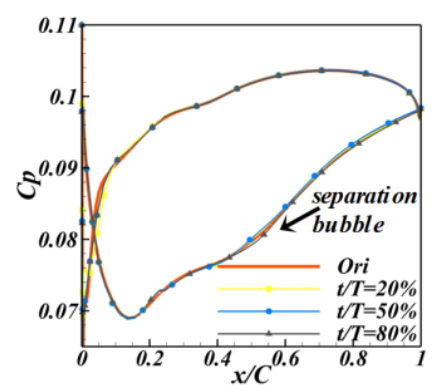

(b) $40 \% \mathrm{H}$ of $\mathrm{BF}$

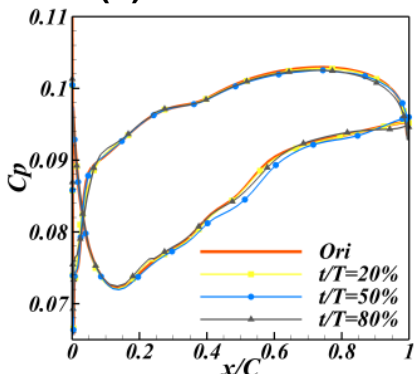

(d) $10 \% \mathrm{H}$ of $\mathrm{BF}$

\section{Figure 7 Distribution of Static Pressure Coefficient at Different Blade Heights}

The values of $\mathrm{Cp}$, distributed at $10 \%$ and $40 \%$ height on the blade surface of FF and EF cascades respectively, is shown in Figure 7. The enclosing area of $\mathrm{Cp}$ curve represents the blade loading. In general, the fence configurations do not affect the blade loading in midspan $(40 \% \mathrm{H})$ but can largely change that near the endwall. The concave at midspan of the datum cascade respects the suction surface separation bubble (shown by the pointers in Figure 7(a) and 7(b)). Compared to the Ori, the case of the 50\% less the pressure gradient and flattens the concave of the separation bubble. In the endwall region, the static pressure of 50\% FF on SS keeps lower until $70 \% \mathrm{C}$, which is nearly as long as the fence configuration, then raises up more quickly than the Ori. As a result, the blade load at $10 \% \mathrm{H}$ becomes higher in the front but lower near TE. The case of $20 \% \mathrm{~T}$ plays less effect on the distribution of $\mathrm{Cp}$ on $\mathrm{SS}$.

In Figure 8, the developments of loss distribution are depicted on several traverse planes, which are vertical to the axial direction and located at $10 \% \mathrm{C}, 30 \% \mathrm{C}, 50 \% \mathrm{C}, 70 \% \mathrm{C}$, $90 \% \mathrm{C}, 110 \% \mathrm{C}, 135 \% \mathrm{C}$, and $160 \% \mathrm{C}$ downstream of the leading edge, respectively. As seen in Figure 8(a), there are three high loss regions (encircled by the iso-line) located according to the endwall boundary layer/CV, $\mathrm{PV}$ and corner separation/CSV, respectively. Compared to the datum cascade, a new loss region appears near the FSS, which larges the endwall boundary layer loss. That because the block effect, played by fence configurations on the endwall crossflow, leads to the momentum wastage of the endwall crossflow. The difference between the FF and BF is: the wake of a fence in FF has longer distance from the outlet in order to dissipate by interacting more with the endwall crossflow. That is obviously shown in $20 \%$ and $50 \%$ cases (in Figure 8 (b), (c), (d), and (e)), the wake of BF still occurs on the outlet plane. However, it is hard to distinguish the fence wake from the corner separation loss in $80 \%$ cases. For 
another remarkable change out of passages of fenced cascades, the high loss regions of PV and corner separation/CSV gather together to change the entire shape of loss.

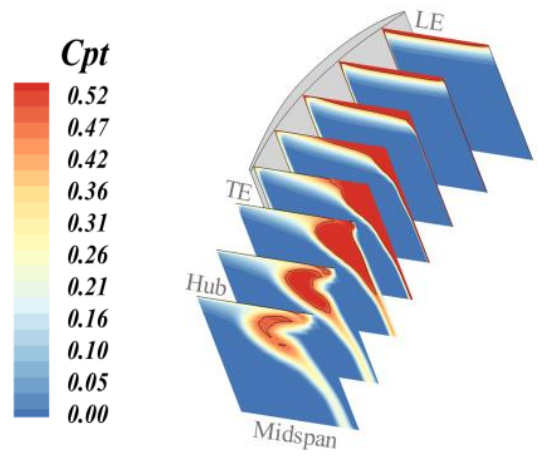

(a) Ori

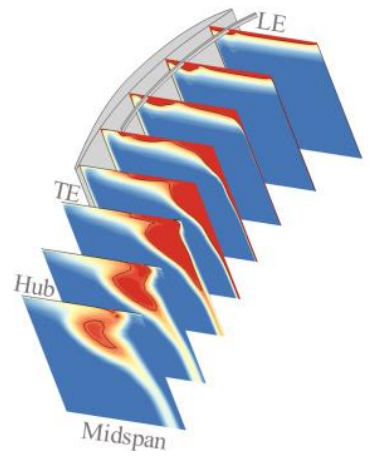

(b) $20 \%$ Case of FF

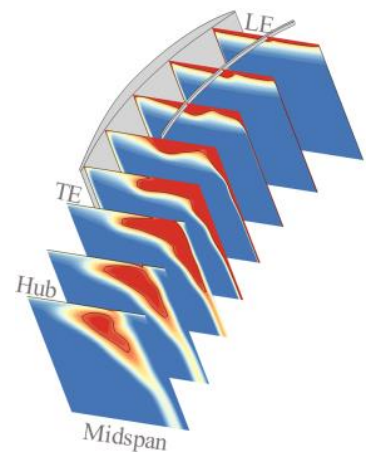

(d) $50 \%$ Case of FF

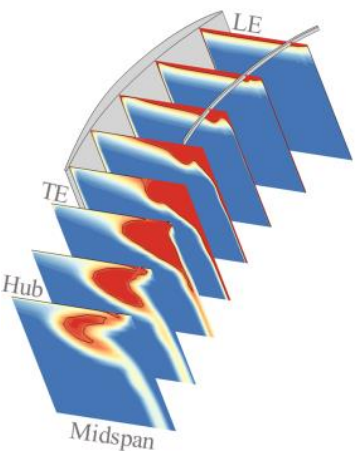

(f) $80 \%$ t Case of FF

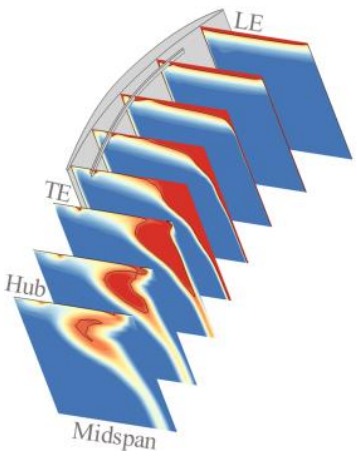

(c) $20 \%$ t Case of BF

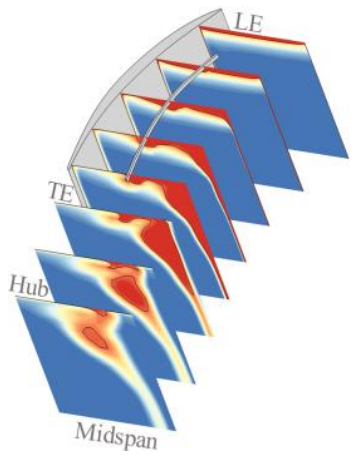

(e) $50 \%$ t Case of BF

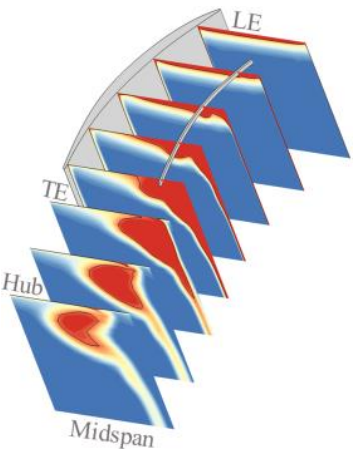

(g) $80 \%$ t Case of BF
Figure 8 Loss Coefficient Contours at Different Axial Sections

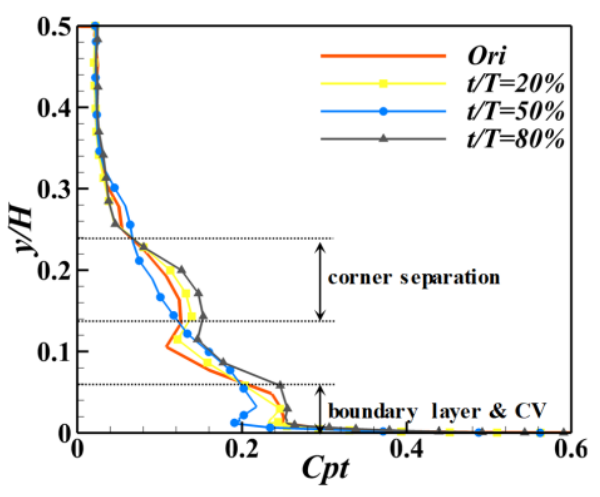

(a) Cases of FF

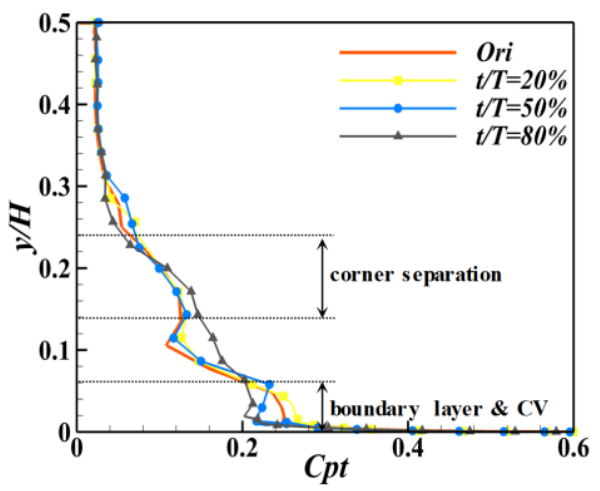

(b) Cases of BF

Figure 9 Spanwise Distribution of Pitchwise Massaveraged Total Pressure Loss Coefficient at Cascade Exit

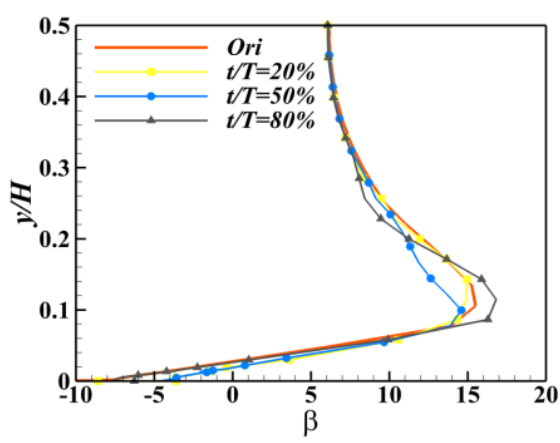

(a) Cases of FF

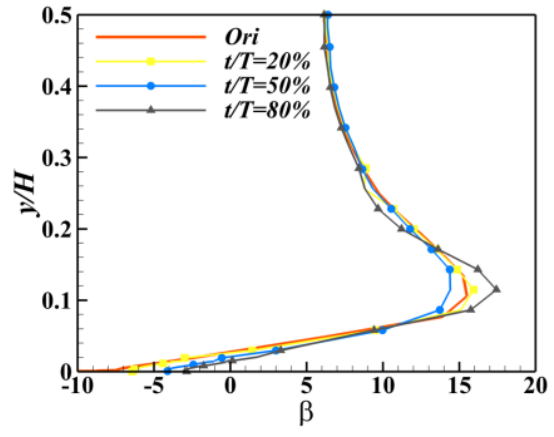

(b) Cases of BF

Figure 10 Spanwise Distribution of Pitchwise Massaveraged Flow Angle at Cascade Exit

The distribution of massflow-average $\mathrm{Cpt}$ along with blade height in Figure 9(a) shows that 50\% T FF can 
simultaneously reduce the loss of the corner separation (between $14 \% \mathrm{H} 24 \% \mathrm{H}$ ) and largely decrease the loss of endwall boundary layer (above $6 \% \mathrm{H}$ ). For this reason, the uniformity along spanwise at cascade exit is raised, which brings a more homogeneous inflow condition to the next cascade. In $\mathrm{BF}$ cases, the $50 \% \mathrm{~T}$ and $80 \% \mathrm{~T}$ fence configurations also effectively decrease the mixing of boundary layer and corner separation (above $6 \% \mathrm{H}$ ), but theirs higher additional loss increases the loss distributed in $5 \% \mathrm{H} \sim 10 \% \mathrm{H}$. In addition, both of the optimized fence cases in the series of FF and BF can the largest extent reduce the value gradient along with the blade height.

The selected cases of FF and BF show the optimal effect of the mass-average outflow angle in Figure 10. Due to the blockage effect fence configurations bringing to the endwall crossflow, the flow angle near the endwall $(0 \% \mathrm{H} 6 \% \mathrm{H})$ is improved by the cases of $20 \% \mathrm{~T} \mathrm{FF}, 50 \% \mathrm{~T} \mathrm{FF}, 50 \% \mathrm{~T} \mathrm{BF}$, and $80 \% \mathrm{~T}$ BF. Reversely, both of the $20 \% \mathrm{~T}$ cases in FF and $\mathrm{BF}$ have less effect on changing the distribution curves of the Ori. Among $8 \% \mathrm{H} 18 \% \mathrm{H}, 80 \% \mathrm{~T}$ cases in $\mathrm{FF}$ and BF play an opposite role against the $50 \% \mathrm{~T}$ cases that reduce the excessive deflection of the outlet flow.

\section{Variations in vortex structure of cascades}

Due to the tiny geometric dimension of fences, the streamlines on SS are influenced by fences weakly (omitted in Figure 11). According to the limiting streamlines of the Ori and two optimal cases (see in Figure 11), the flow field near the endwall in fenced cascades becomes more complicated than that in the datum cascade. The emergency of the separation lines around the fence configurations is related with the boundary layer separation on the fence surface, which is the outcome of the blockage of strong crossflow by fences. Meanwhile, the crosswise size of the reserve flow in corner region is extended: in the FF case, the reserve flow from FPS intrudes the corner separation region and induces a clockwise spiral point; in the back case, the counter-clockwise spiral lines occur in the original reserve flow region. That indicates the block effect of fence arrays can weaken the endwall crosswise pressure gradient, which makes it hard to withstand the low-energy flow from PS with a higher pressure. Last but not least, the back case has a more extended wake of the fence than the front case, which would play a negative effect on the downstream cascade.

In Figure 12, the contours of streamwise vorticity and the developments of secondary streamlines are depicted on several traverse planes. In the endwall region of the datum cascade, the positive vorticity in red is caused by the boundary layer as well as the PV, and the blue area with negative vorticity is related to the $\mathrm{CV}$. In the region near SS, the negative vorticity represents the CSV and the positive areas in flow passage are corresponding to the WV. The secondary streamlines show that the PV, the CSV and the CV play the most critical role in the vortex structure outside the Ori flow passage. In a word, the optimal fences make the endwall flow structure better. For the vorticity, the fence configurations bring positive vorticity to counteract the endwall boundary layer by means of the FV, which is caused by the interaction of the fence surface and the crossflow. As a result, the area of endwall negative vorticity is reduced. For the secondary streamlines, the core of the PV is trapped by fences, so it fails to participate in corner separation region. Hence the $\mathrm{CV}$, induced by the PV in $50 \% \mathrm{~T}$ of FF cascade, dissipate in the two optimal fenced cascades when that leave away the TE.

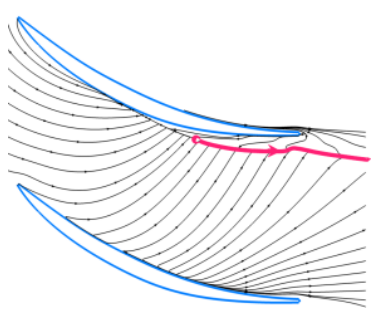

(a) Ori

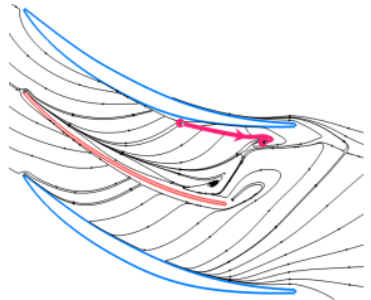

(b) $50 \% \mathrm{~T}$ Case of FF

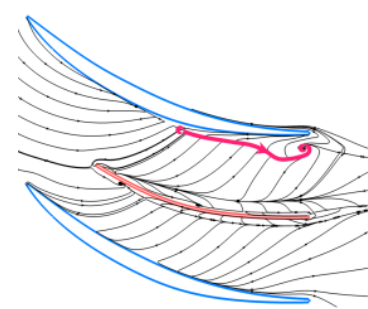

(c) $50 \% \mathrm{~T}$ Case of BF

\section{Figure 11 Limiting Streamlines on the Endwall}

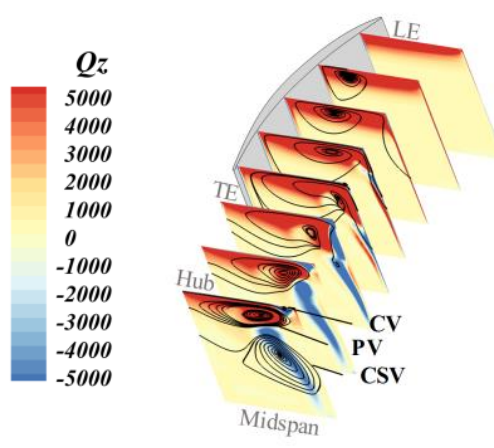

(a) Ori

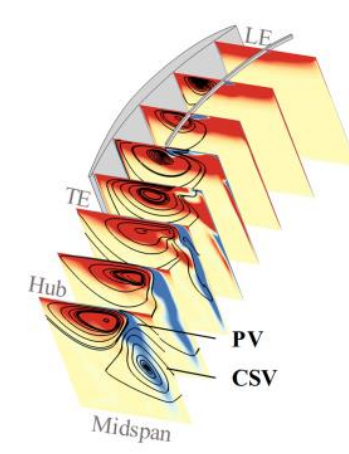

(b) $50 \% \mathrm{~T}$ Case of $\mathrm{FF}$

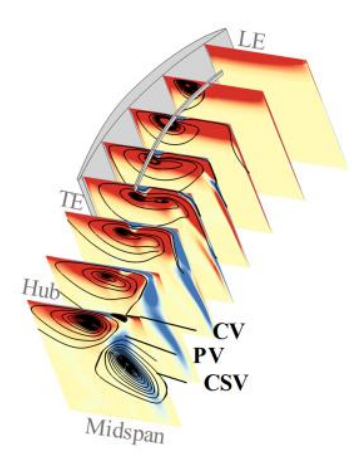

(c) $50 \% \mathrm{~T}$ Case of BF

\section{Figure 12 Distribution of Axial Vorticity and Secondary Streamlines in Blade Passage}

Figure 13 shows the secondary flow behaviour visualized by the iso-surface of Q-criteria, which is colored 
by vorticity. For the datum cascade, the endwall crossflow transport from the LE of the PS to the corner separation, and the PV, the CSV and the CV mainly organize the flow field exit the passage. Optimal fences block the transportation of crossflow between fences and the PS, and finally reduce the corner separation. Besides that, the PV, of which crosswise actuating range is decreased, directly cause the development of the CV: the CV hard to resist the high-energy flow from the trailing edge of the PS. Therefore, the CV in fenced cascades dissipates in the faster speed and end up earlier.

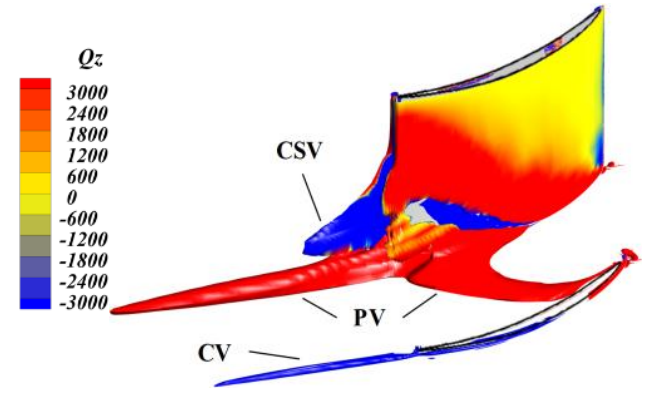

(a) Ori

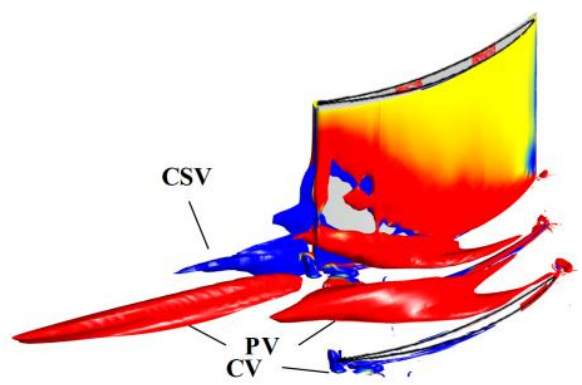

(b) $50 \% \mathrm{~T}$ Case of FF

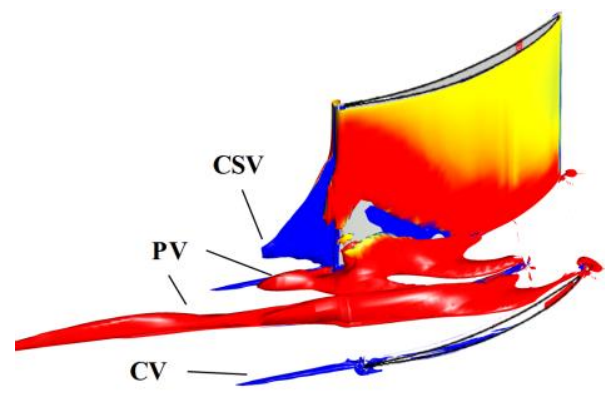

(c) $50 \% \mathrm{~T}$ Case of $\mathrm{BF}$

\section{Figure 13 3D Vortex Structure in Flow Passage}

\section{Variations in inflow incidences of cascades}

Figure 14 shows the $\mathrm{Cpt}$ for the datum and the optimum case under six different incidences. At the design Mach number, it is clear that Cpt increases with leaving away from the design point. Compared to the curve of the Ori, the fenced case has no reduction in $\mathrm{Cpt}$ attained in all positive incidences and $0^{\circ}$. For negative incidences, the optimal fence array has stronger control effect with the decrease of incidence. The highest reduction $(-29.7 \%)$ is reached where the incidence is $-9^{\circ}$. Consequently, optimization of the endwall fence configurations to be designed to cover a broader range of operation is still needed.

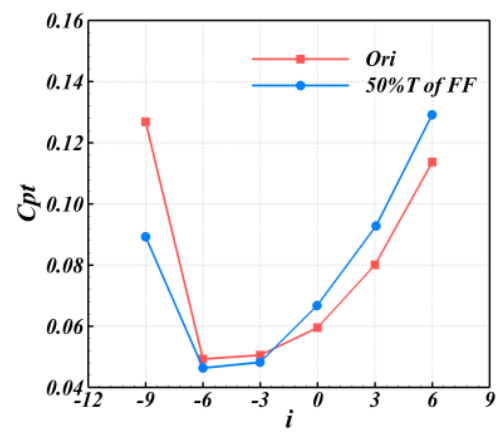

\section{Figure 14 Total Pressure Loss Coefficient Versus Incidence Angle}

\section{CONCLUSIONS}

In this study on aerodynamic performance and vortex structure of high-speed compressor cascade with varying locations of fence configurations, numerical analyses are used to derive the following points:

1. At the design point, both the cases of $50 \% \mathrm{~T} \mathrm{FF}$ and $\mathrm{BF}$ have better aerodynamic performance. The $50 \% \mathrm{~T}$ FF can reduce the total pressure loss coefficient and blockage coefficient by $6.5 \%$ and $6.9 \%$, respectively, and increases the absolute value of turning angle by $0.9 \%$. For BF, the $50 \% \mathrm{~T}$ plays better on the reduction of loss and blockage.

2. Both optimal cases of $\mathrm{FF}$ and $\mathrm{BF}$ can reduce the loss in the endwall region $(0 \% \mathrm{H} \sim 6 \% \mathrm{H})$ and the excessive deflection of flow angle in $8 \% \mathrm{H} 18 \% \mathrm{H}$. Hence the spanwise uniformity of aerodynamic parameters at the exit is raised.

3. The optimum case brings a positive effect on the cascade by blocking endwall crossflow, delaying the transportation of the $\mathrm{PV}$, and reducing the vorticity of endwall crossflow effectively.

4. The secondary flow on the outlet plane of this highspeed compressor cascade is mainly composed of the PV, the $\mathrm{CSV}$, and the CV. The 50\% $\mathrm{T}$ FF case, as the optimum endwall fence, not only weakens the PV but also dissipates the $\mathrm{CV}$ before the cascade exit, which simplifies the vortex structure and reduces the associated secondary flow loss.

5. Compared to the datum cascade and the optimum cascade, a reduction of the loss exists at negative incidences while an augment occurs at positive incidences. The optimal endwall fence is limited to enlarge the cascade operating range under negative incidences.

\section{NOMENCLATURE}

$\begin{array}{ll}\text { Notation } & \\ \mathrm{i} & \text { inflow incidence } \\ \mathrm{x} & \text { spanwise coordinate } \\ \mathrm{y} & \text { pitchwise coordinate } \\ \mathrm{y}+ & \text { nondimensional distance from wall } \\ \mathrm{z} & \text { streamwise coordinate } \\ \mathrm{A} & \text { azimuthal cross section } \\ \mathrm{B} & \text { blockage coefficient } \\ \mathrm{C} & \text { blade chord length } \\ \mathrm{H} & \text { blade span }\end{array}$




$\begin{array}{ll}\text { Ma } & \text { Mach number } \\ \text { P } & \text { pressure } \\ \text { Q } & \text { vortex criterion } \\ \text { T } & \text { pitch } \\ \text { V } & \text { velocity } \\ \text { Greek } & \\ \beta & \text { flow angle } \\ \delta & \text { inflow boundary layer thickness } \\ \rho & \text { density } \\ \Omega & \text { vorticity } \\ \text { Subscripts } & \\ \text { b } & \text { blockage region } \\ \text { e } & \text { the edge of defect region } \\ \text { in } & \text { inlet parameter } \\ \text { out } & \text { outlet parameter } \\ \text { s } & \text { secondary component } \\ \text { t } & \text { stagnation flow parameter } \\ \text { Abbreviations } & \\ \text { BF } & \text { back fence(s) } \\ \text { Cp } & \text { static pressure coefficient } \\ \text { Cpt } & \text { total pressure loss coefficient } \\ \text { CSV } & \text { concentrated shedding vortex } \\ \text { CV } & \text { corner vortex } \\ \text { FF } & \text { front fence(s) } \\ \text { FPS } & \text { fence pressure side } \\ \text { FSS } & \text { fence suction side } \\ \text { FV } & \text { fence vortex } \\ \text { LE } & \text { leading edge } \\ \text { Ori } & \text { datum cascade } \\ \text { PS } & \text { pressure surface } \\ \text { PS } & \end{array}$

\section{ACKNOWLEDGMENTS}

This study supported by a project funded by the National Natural Science Foundation of China (Grant Nos. 51506020 and 51436002).

\section{REFERENCES}

Chen P. P. et al. (2012) Passive control of hub-corner separation/stall using axisymmetric-hub contouring, Proceedings of the Institution of Mechanical Engineers, Part G: Journal of Aerospace Engineering, 226(10), pp. 12141224. doi: 10.1177/0954410011421716.

Denton J. D. (1993) Loss Mechanisms in Turbomachines, in, p. V002T14A001. doi: 10.1115/93-gt435.

Diaa A. M. et al. (2015) Boundary layer control of an axial compressor cascade using nonconventional vortex generators, ASME International Mechanical Engineering
Congress and Exposition, Proceedings (IMECE), 1-2015, pp. 1-9. doi: 10.1115/IMECE2015-52310.

Gad-el-Hak M. (1996) Modern Developments in Flow Control, Applied Mechanics Reviews, 49(7), p. 365. doi: 10.1115/1.3101931.

Gbadebo S. A., Cumpsty, N. A. and Hynes, T. P. (2005) Three-dimensional separations in axial compressors, Journal of turbomachinery, 127(2), pp. 331-339.

Govardhan M. and Kumar, K. N. (2011) Secondary flow loss reduction in a turbine cascade with a linearly varied height streamwise endwall fence, International Journal of Rotating Machinery, 2011(August 2015). doi: 10.1155/2011/352819.

Hage W. and Paschereit, C. O. (2007) Control of Secondary Flow in a High Loaded Compressor Stage by Means of a Groove Structure on the Sidewalls, (June), pp. 112.

Hecklau M. et al. (2012) Active Separation Control with Pulsed Jets in a Critically Loaded Compressor Cascade, AIAA Journal, pp. 1729-1739. doi: 10.2514/1.j050931.

Hergt A. et al. (2011) A New Approach for Compressor Endwall Contouring, ASME Turbo Expo 2011, 1, pp. 1-10. doi: 10.1115/GT2011-45858.

Kawai T. (1994) Effect of Combined Boundary Layer Fences on Turbine Secondary Flow and Losses., JSME International Journal Series B, 37(2), pp. 377-384. doi: 10.1299/jsmeb.37.377.

Li Y. H. et al. (2010) Control of the corner separation in a compressor cascade by steady and unsteady plasma aerodynamic actuation, Experiments in Fluids, pp. 10151023. doi: 10.1007/s00348-009-0787-2.

Liu H. et al. (2017) Secondary flow control using endwall jet fence in a high-speed compressor cascade, Journal of Mechanical Science and Technology, 31(10), pp. 4841-4852. doi: 10.1007/s12206-017-0932-4.

Moon Y. J. and Koh S. R. (2001) Counter-rotating streamwise vortex formation in the turbine cascade with endwall fence, Computers and Fluids, 30(4), pp. 473-490. doi: 10.1016/S0045-7930(00)00026-8.

Zander V. et al. (2011) Active flow control by means of synthetic jets on a highly loaded compressor cascade, Proceedings of the Institution of Mechanical Engineers, Part A: Journal of Power and Energy, 225(7), pp. 897-906. doi: 10.1177/0957650911410275.

Zhang H. D. et al. (2018) Flow control using unsteady pulsed holed suction with different excitation models in a highly loaded compressor cascade, Proceedings of the Institution of Mechanical Engineers, Part A: Journal of Power and Energy. Elsevier Masson SAS, 232(6), pp. 593607. doi: 10.1177/0957650917740769.

Zhong J. J. et al. (2009) Numerical Simulation of Endwall Fence on the Secondary Flow in Compressor Cascade, in, pp. 509-518. doi: 10.1115/gt2008-50888. 